\title{
Axillary web syndrome following breast cancer surgery: symptoms, complications, and management strategies
}

This article was published in the following Dove Press journal: Breast Cancer - Targets and Therapy

\section{LA Koehler ${ }^{1-3}$ \\ TC Haddad ${ }^{4}$ \\ DW Hunter ${ }^{5}$ \\ TM Tuttle T.6 $^{3,6}$}

'Division of Physical Therapy, ${ }^{2}$ Division of Rehabilitation Medicine, Department of Rehabilitation Medicine, Medical School, University of Minnesota, Minneapolis, MN, USA; ${ }^{3}$ University of Minnesota, Masonic Cancer Center, Minneapolis, MN, USA; ${ }^{4}$ Mayo Clinic, Division of Medical Oncology, Department of Oncology, Rochester, MN, USA; ${ }^{5}$ Department of Radiology, Medical School, University of Minnesota, Minneapolis, MN, USA; ${ }^{6}$ Department of Surgery, Medical School, University of Minnesota, Minneapolis, MN, USA
Correspondence: LA Koehler Division of Physical Therapy, Department of Rehabilitation Medicine, University of Minnesota Medical School, Mayo Mail Code 388,420 Delaware St. SE, Minneapolis, MN 55455, USA

$\mathrm{Tel}+\mid$ 6I2 $626 \mid 502$

Fax + I 6126254274

Email koeh0139@umn.edu

\begin{abstract}
Axillary web syndrome (AWS) is a common condition occurring in up to $86 \%$ of patients following breast cancer surgery with ipsilateral lymphadenectomy of one or more nodes. AWS presents as a single cord or multiple thin cords in the subcutaneous tissues of the ipsilateral axilla. The cords may extend variable distances "down" the ipsilateral arm and/or chest wall. The cords frequently result in painful shoulder abduction and limited shoulder range of motion. AWS most frequently becomes symptomatic between 2 and 8 weeks postoperatively but can also develop and recur months to years after surgery. Education about and increased awareness of AWS should be promoted for patients and caregivers. Assessments for AWS should be performed on a regular basis following breast cancer surgery especially if there has been associated lymphadenectomy. Physical therapy, which consists of manual therapy, exercise, education, and other rehabilitation modalities to improve range of motion and decrease pain, is recommended in the treatment of AWS.
\end{abstract}

Keywords: cording, Mondor's disease, rehabilitation, axillary lymphadenectomy

\section{Introduction}

Axillary web syndrome (AWS) is a common but often overlooked condition that most commonly occurs in patients following breast cancer surgery with axillary lymph node dissection (ALND). ${ }^{1-4}$ It also occurs in patients with other shoulder and axillary pathology, including trauma, infection, sentinal node lymphadenectomy, ${ }^{5}$ and melanoma surgery with axillary lymphadenectomy. ${ }^{6}$ In the literature, various synonyms for AWS have included cording, lymphatic cording, fibrous banding; and incorrectly, Mondor's disease. AWS presents as a "tight" cord in the subcutaneous tissue in the axilla. ${ }^{3}$ The cord may extend down the medial or medial-volar surface of the ipsilateral upper arm and/or down along the ipsilateral lateral chest wall. The cord tightens, often painfully, with shoulder abduction. AWS most commonly develops 2-8 weeks following breast cancer surgery. ${ }^{1-4,7}$ However, recent studies have shown that AWS can occur months to years later and can also resolve and then relapse. ${ }^{2,7}$

The reported incidence of AWS ranges from $6 \%$ to $86 \%$ following breast cancer surgery. The wide range arises from several reasons, the most important of which appears to be whether or not AWS was specifically sought on postoperative examinations. The incidence is also dependent on the type of surgery and the length of follow-up..$^{1-4,7-10}$ Two recent careful prospective studies that included evaluations of postoperative breast cancer patients demonstrated that the actual value may lie somewhere between these two extremes. One study had a prevalence of AWS of $50 \%$ at 18 months, ${ }^{2}$ and the other had a $51 \%$ incidence in the 8 weeks ${ }^{11}$ following surgery. The 
incidence is higher in surgery with ALND $(36 \%-72 \%)^{1,2,4,7,9,10}$ compared with surgery with sentinel lymph node biopsy (SNB) $(11 \%-58 \%))^{1,4,7,10}$ For reasons that are not clear, the incidence of AWS is highest in patients who have a prior or contemporaneous contralateral prophylactic mastectomy $(86 \%){ }^{2}$ The incidence is also higher in patients who have a lower body mass index, ${ }^{1,10,12}$ who are younger age, ${ }^{1,8,10,11}$ who have more education, ${ }^{11}$ who exercise more frequently, ${ }^{11}$ who have a greater number of lymph nodes removed, ${ }^{1,7}$ who receive more extensive surgery, ${ }^{1,7,11}$ or who receive adjunctive chemotherapy $^{7}$ or radiation therapy. ${ }^{7}$

In our local experience, AWS is currently getting diagnosed in the Breast Cancer Center by dedicated physical therapists doing research on AWS. AWS had previously been diagnosed less frequently than currently because providers and patients were unfamiliar with the signs and symptoms. In addition, some providers who were knowledgeable about AWS did not refer their patients for evaluation because they were unaware of the treatment options available from physical therapy. The primary focus of care following surgery is on cancer treatment planning, not on the less threatening physical impairments of conditions like AWS. Patients see multiple providers after surgery including surgeons, radiation oncologists, medical oncologists, and primary care providers making it difficult for the patient to know to whom they should report their symptoms and to whom they should turn for advice on how to manage their symptoms. Providers are each addressing symptoms with which they are familiar and may assume others are assessing and addressing less pressing physical symptoms such as AWS. The purpose of this article is to describe the signs and symptoms, diagnosis and management, and potential complications of AWS.

\section{Signs and symptoms}

AWS presents as a tight, $\sim 1 \mathrm{~mm}$ wide, linear singular cord or multiple cords of tissue in the subcutaneous tissue of the axilla (Figure 1). The cords may extend "down" the medial or medial-volar ipsilateral upper arm (Figure 2) or "down" along the lateral edge of the ipsilateral chest wall. The cords become visible and/or palpable when the arm is fully extended (straight) and then abducted. One study suggested that in $>50 \%$ of patients the cords will not be visible requiring careful palpation to detect it. ${ }^{1}$ Another study indicated that $>70 \%$ of cords are palpable, with the implication being that the remainder were only visible. ${ }^{13}$ If the arm is straightened at the elbow and then abducted adequately essentially all cords are palpable and many are visible as a linear "tenting" or "furrowing" of the skin. When the arm is not in the "straightened" (elbow extended) and abducted position, tension is taken off the cord, and the cord may not be evident.

AWS is usually diagnosed $2-8$ weeks following breast cancer surgery. ${ }^{2,3,6,7}$ A recent prospective study ${ }^{11}$ suggested that $94 \%$ of patients who developed AWS diagnosed it within the first 4 weeks using a self-assessment tool. Another prospective study ${ }^{13}$ found that careful practitioner evaluation was able to detect $66 \%$ of AWS cases within 7 days of surgery. In addition, AWS can present months to years later ${ }^{2,7,8,14,15}$ and may also reoccur after resolution. ${ }^{2}$ Therefore, assessment for AWS should be performed on a frequent basis in the first 3-6 months after surgery, and then less frequently but still regularly for up to 3 years.

At the onset of AWS, patients experience restricted upper extremity movement, primarily during shoulder abduction, and pain or discomfort whenever arm movement increases the tension on the cord. Patients who have not been educated about AWS may be unaware that they have developed a cord. In our experience, patients in whom the diagnosis was delayed often believed that their symptoms were a "normal" part of the postoperative recovery. The full gamut of patient symptoms is dependent on the cord location. Some patients may be unable to extend their elbow because the cord extends across the antecubital fossa. In these cases, the patients may come to the clinic with their arm adducted across their trunk in a sling-like position. Cord extension below the elbow occurs more commonly in patients who have had ALND compared with those who have had SNB. ${ }^{4}$

Patients with less rigid or shorter cords may have minimal cord tension and minimal symptoms until they approach full extension and abduction. In our experience, they frequently describe arm movement as feeling "different" or "not nor-

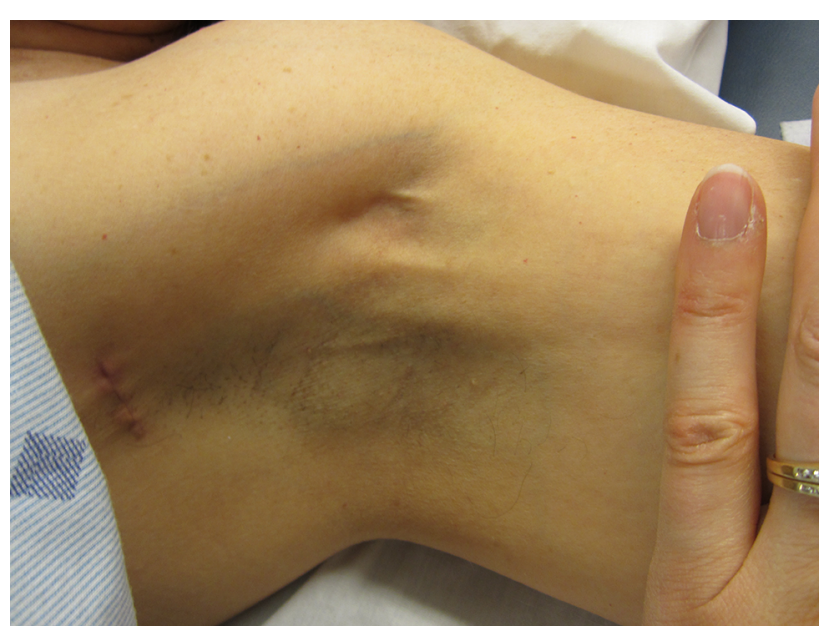

Figure I Axillary web syndrome of the left axilla. Note: Multiple cords are visible in the mid axilla. 


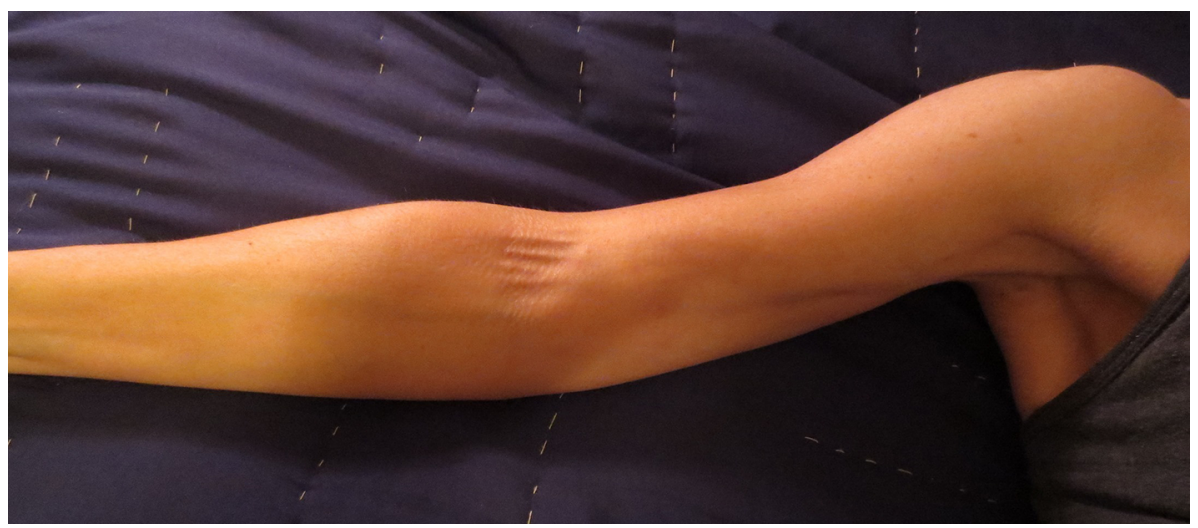

Figure 2 Axillary web syndrome of the right extremity.

Note: Multiple cords are visible in the axilla and medial upper extremity with extension into the antecubital fossa.

mal" but their movements are not painful and they are often unaware of the cord. We have seen patients who "learned to live" with their symptoms and later developed chronic shoulder mobility and strength issues.

On occasion, subcutaneous nodules may appear to be connected to AWS cords. Two studies have addressed the concern that the nodules represent metastatic disease. ${ }^{16,17}$ Biopsies of the nodules demonstrated enlarged lymph vessels ${ }^{16}$ and lymph vessels surrounded by fatty tissue. ${ }^{17}$ It was speculated that the nodules are due to engorgement of the obstructed lymphatic vessels. At this time, no postoperative AWS cord-related nodules have histologically demonstrated metastatic disease.

AWS has been reported to occur prior to breast cancer surgery, likely due to lymphatic involvement by metastatic disease. ${ }^{5}$ In this study, the patients had subsequent documentation of metastatic spread to axillary lymph nodes. The authors speculated that an aggregation of cancer cells leads to lymphatic obstruction that causes elevated pressures similar to the pressure increase due to surgical interruption. The pathophysiological explanation for preoperative AWS remains a hypothesis, but based on our experience and the literature, it seems prudent to consider the possibility of metastatic spread in making decisions about patient management.

\section{Complications}

Early literature described AWS as a self-limited condition, which resolved within 3 months of onset. ${ }^{3,4}$ More recent research has demonstrated that AWS does not resolve in all patients, can persist for years after surgery, and may reoccur after resolution. ${ }^{1,2,7}$ AWS development has been associated with early and delayed physical complications following breast cancer surgery. ${ }^{1,2,7}$ Patients with AWS experience statistically lower range of motion for shoulder abduction and flexion compared with patients without AWS. ${ }^{1}$ Reduced function and lymphedema have also been associated with AWS. ${ }^{2,7}$ The actual incidence of lymphedema in patients with AWS is lower than we initially expected and from a clinical standpoint some recent studies suggest that there may be no direct correlation between the presence of AWS and the development of lymphedema. In one study, ${ }^{18} 35.9 \%$ of their surgical patients developed AWS and 31.4\% developed lymphedema. However, the overlap was small enough that there was no statistically significant association between AWS and the development of lymphedema (OR $=0.87,95 \%$ CI $0.65-1.15, P=0.329)$. In the other study, ${ }^{19}$ the AWS group had less increase in upper arm volume than the non-AWS group in which upper extremity volumes were increased. Overall, the AWS group had significantly more pain, less active ROM in shoulder abduction, and a lower upper-limb volume at $0-10 \mathrm{~cm}$ proximal to the lateral epicondyle. The overall incidence of lymphedema was $9.9 \%$ and was not associated with AWS. In our experience following AWS patients for longer than 2 years, it appears that chronic AWS may lead to other secondary problems such as chronic alteration of movement patterns and shoulder dysfunction and pain. This has been substantiated in some other recent studies ${ }^{2,19,20}$ but further research is needed to determine the scope and severity of complications associated with AWS. One recent study ${ }^{21}$ found non-specific vascular changes in the affected arm, the significance of which are currently unknown.

Receiving a breast cancer diagnosis and undergoing breast cancer treatment is very stressful for patients. In our experience, AWS may lead to a higher level of psychological distress and anxiety due to the additional burdens of loss of function, reduced movement, and pain. Some patients have also related fears that the symptoms might be a sign of cancer recurrence. The small amount of research has been 
done on this matter ${ }^{5,7}$ has not found any association between postoperative AWS and cancer recurrence. Patient education and physical therapy treatment for AWS may help to reduce overall levels of patient anxiety.

\section{Assessment}

Assessment for AWS does not usually require further testing beyond physical examination to determine if AWS is present or not present (Figure 3A). Description of the location of AWS (axilla, upper arm, elbow, lower arm, wrist/hand, lateral chest wall) and the number of cords present (single/ multiple) are also beneficial (Figure 3B).

A comprehensive assessment for AWS was established for research purposes ${ }^{22}$ but could also be used in clinical practice to objectively assess AWS. The length, width, and depth of each cord can be measured at each location to assess change over time. ${ }^{22}$ A tape measure can be used to measure the length of
AWS cords (in cm) but it is difficult to measure the width and depth with a tape measure, therefore subjective judgment can be used to assess width $(<,>$, or $=$ to $1 \mathrm{~mm}$ ) and depth (superficial, middle, deep). Using a marking pen to outline the cord on the surface of the skin will allow the cords to be measured more easily if the cords are palpable but not visible. ${ }^{22}$ Pictures are also beneficial to document changes. Goniometric shoulder movements (active and passive range of motion) should be used to assess for movement restrictions with specific attention to shoulder abduction as it is the most affected movement followed by flexion. ${ }^{1}$ Elbow and hand motion should also be measured if affected. Physical therapists, who are movement specialists, can provide a thorough shoulder assessment on postural changes and shoulder kinematics, including scapular dysfunction and altered movement patterns. Location and intensity of pain should be assessed during upper extremity movements since tension on the cord occurs during movement especially shoulder
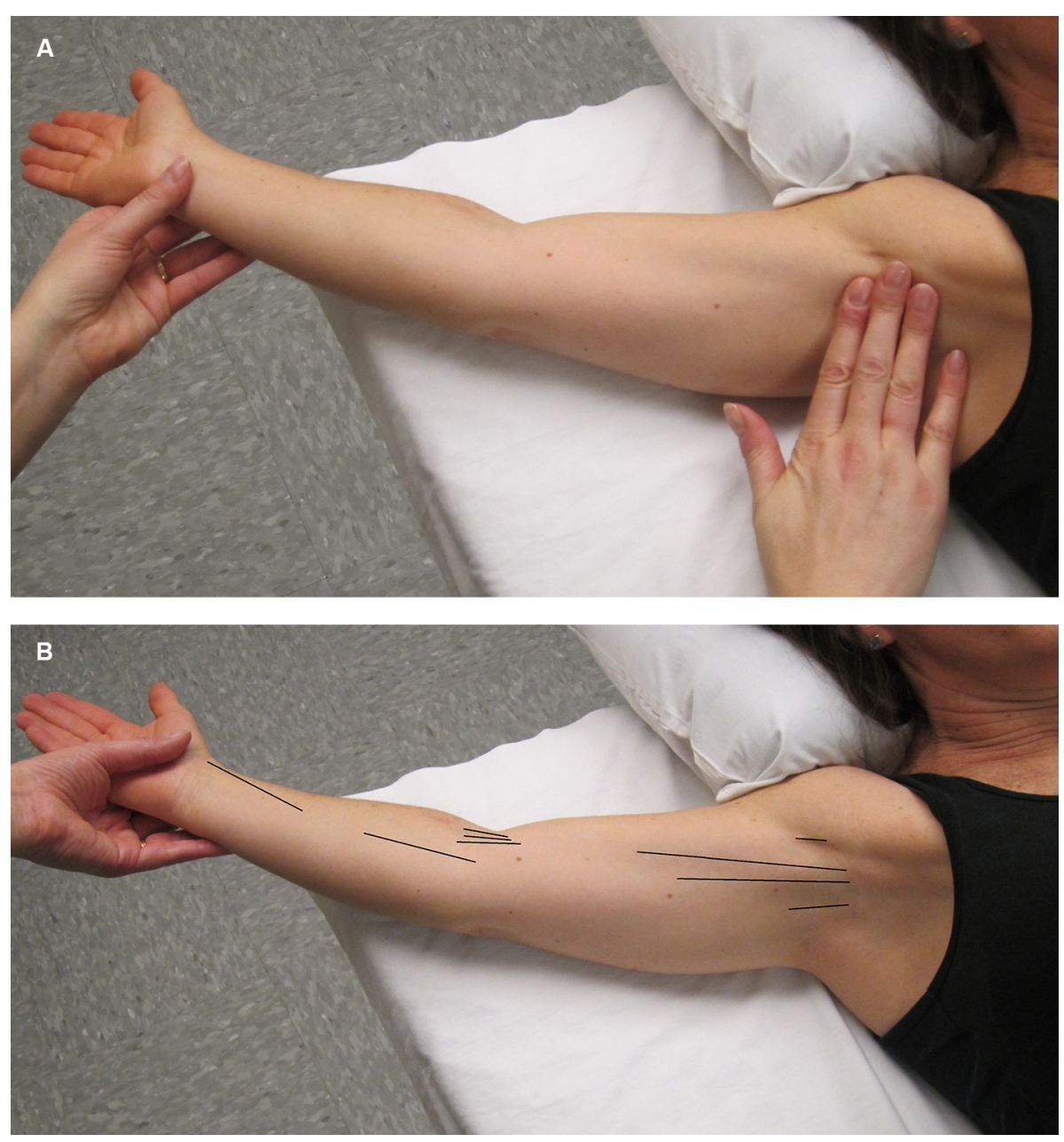

Figure 3 (A) In order to make an accurate diagnosis of the presence or absence of AWS. The physical examination should be performed in a manner that is designed to facilitate the search for the problem. The first step is to gently but maximally extend the arm at the elbow and then gently but maximally abduct the affected arm at the shoulder. The person performing the evaluation both visualizes and palpates for cords in the locations indicated in (B). (B) This figure illustrates the locations (see black lines) in which a cord or cords may be found, including the axilla, down the upper arm from the axilla to and across the antecubital space, and rarely down the forearm to the base of the thumb.

Abbreviation: AWS, axillary web syndrome. 
abduction. The Disabilities of the Arm, Shoulder, and Hand questionnaire has been frequently used to assess upper extremity shoulder function in individuals with $\mathrm{AWS}^{1,2,7,20,22}$ but other questionnaires may also be useful to assess pain, function, and/ or quality of life in individuals with AWS., ${ }^{2,711,20}$ Lymphedema assessment is also warranted ${ }^{1,2,7,8,15,20}$ using available measuring methods such as girth measurements, perometer, bioimpedance, and/or tissue dielectric constant.

Although rare, there are cases, listed below, in which further testing may be indicated to rule out other potential causes of cording.

- Chest wall cording: AWS-related cording on the chest wall may be difficult to detect and differentiate from Mondor's disease (or syndrome). When cords are located on the chest wall, some doctors appear to consider AWS and Mondor's disease synonymous. ${ }^{23}$ Mondor's disease is a thrombosis of a superficial chest wall vein. Mondor's disease is often described as having a linear or curvilinear vessel-like appearance with occasional redness or swelling, which may appear as a protuberance or furrow, and is often thicker than the AWS cord. Mondor's disease may be present following breast augmentation and is often located along the inferior fold of the breast. In our experience, high frequency ultrasound $(15 \mathrm{MHz}$ or greater) or high resolution MR imaging is able to reliably determine if the band is a thrombosed superficial vein. If either of these two imaging tests fail to find a vascular structure, our results and those of one other study indicate that the cord is due to AWS since AWS is not associated with a consistent pathological structure using ultrasound imaging. ${ }^{24,25}$

- AWS with nodules: Currently, there is no evidence that nodules attached or adjacent to AWS cords following surgery are associated with metastatic disease. Nonetheless, we would agree that a biopsy of the nodule should be considered if there is any concern for potential metastatic disease.

- AWS prior to cancer surgery: Cases of AWS prior to breast surgery may be a sign of metastatic disease to the lymph nodes..$^{5}$ A thorough examination of the axillary lymph nodes should be performed prior to or during surgery. Additionally, if it is felt that it may impact the type or extent of the surgery preoperative MRI or axillary ultrasonography can be used to identify and potentially biopsy suspicious lymph nodes.

- AWS unrelated to cancer surgery: Individuals who experience AWS unrelated to cancer surgery may need to undergo further testing to determine the cause of the condition.
Some cases of AWS have been related to infection and strenuous activity. ${ }^{5,26}$ If there is any concern about metastatic disease involving the axillary lymph nodes, diagnostic imaging such as PET-CT, mammography, ultrasound, or MRI of the breast and axilla should also be considered.

\section{Treatment strategies}

Based on our experience and results from several other studies, ${ }^{4,14,15,17,20,27-31}$ physical therapy is recommended as a safe and effective primary treatment for AWS. These studies support the hypothesis that physical therapy resolves cording more rapidly than no treatment. Physical therapy treatment at our institution consists of an initial process of patient education, supervised and at-home exercises and tissue "manipulations" including a variety of adjunctive rehabilitation interventions to improve range of motion and decrease pain, and therapist-performed, in-clinic manual therapy including myofascial release, soft tissue mobilization, and cord manipulation and stretching while the arm is abducted. Nonsteroidal anti-inflammatory drugs and opioids have also been recommended based on the severity of associated pain. ${ }^{32}$ If, as we currently believe, AWS is due to lymphatic pathology, treatment by a physical therapist specialized in lymphedema and/or cancer rehabilitation would be advantageous. Other physical therapy techniques that have a positive effect on the outcome of AWS include compression bandaging and manual lymphatic drainage. Scar release manipulation and massage of adhesions can assist with releasing the "adhesive" tissue that seems to be an integral part of the AWS cords. Joint mobilization can lessen secondary joint restrictions in the shoulder, scapula, rib, clavicle, and upper back. Stretching and strengthening exercises can improve range of motion and muscle strength. In those patients with the capability and understanding, the at-home program can even be expanded to include the exercises and manual techniques directed at the cord and associated adhesions mentioned above.

If AWS is accompanied by lymphedema, physical therapy utilizing adjunctive manual lymphatic drainage has been shown to significantly reduce arm volume and pain compared with physical therapy alone. ${ }^{20}$ Cords that extend into the extremity have also been reported to resolve with short-term use (1-2 days) of gradient compression lymphedema bandaging of the extremity. ${ }^{30}$ Therapists have reported feeling a pop or snap in the cord during manipulative or massage treatment. The sensation feels to the therapist as if the cord is breaking. ${ }^{17,28}$ The problem has not been studied adequately to determine if "breaking a cord" is safe or effective in the "long term". In the anecdotal experience at our institution, breaking of the cord 
is usually followed by a sudden increase in mobility and a decrease in the tension of the cord resulting in partial symptom resolution. It has been speculated that the supporting fibrous or inflammatory tissue that surrounds the cord might be breaking and not the cord itself. ${ }^{28}$ Our short-term experience indicates that "breaking" a cord does not have any negative effects such as swelling. The intermediate-term effects of "breaking" a cord indicate that the immediate increase in range of motion tends to be maintained. But, if the pathophysiologic basis for the AWS cords is pathology of the lymphatic vessels, such as leakage due to upstream obstruction followed by extra-lymphatic inflammatory or adhesive changes, it is uncertain what effect the "breaking" of the cords might have on the lymphatic system. In general, we feel that gentle manual techniques are recommended. It has also been theorized that AWS cord development could be associated with lymphangiogenesis., ${ }^{5,15,22}$ If this is true, it is another reason that aggressive manual techniques may not be appropriate.

\section{Conclusion/summary}

AWS is a common condition following breast cancer surgery with ALND. It usually presents within 2-8 weeks of surgery but can develop or recur months to years later. It can be associated with later lymphedema in a minority of patients. It presents as a tight subcutaneous cord in the ipsilateral axilla. The cord becomes increasingly evident and tense as the shoulder is abducted. Patients complain of pain with abduction and limited function and range of motion in the affected extremity. Physical therapy and exercise can reduce pain and increase range of motion. Education about and awareness of AWS should be promoted for both patients and providers, and regular assessments for AWS should be initiated by health care providers.

\section{Acknowledgment}

The authors would like to thank Mackenzie Dwyer for her assistance in preparing the manuscript and Elena MacDonald and Heather Thompson Buum for their contributions.

\section{Disclosure}

The authors report no conflicts of interest in this work.

\section{References}

1. Koehler LA, Blaes AH, Haddad TC, Hunter DW, Hirsch AT, Ludewig PM. Research report movement, function, pain, and postoperative edema in axillary web syndrome. Phys Ther. 2015;95(10):1345-1353.

2. Koehler LA, Hunter DW, Blaes AH, Haddad TC. Function, shoulder motion, pain, and lymphedema in breast cancer with and without axillary web syndrome: an 18-month follow-up. Phys Ther. 2018;98(6):518-527.
3. Moskovitz AH, Anderson BO, Yeung RS, Byrd DR, Lawton TJ, Moe RE. Axillary web syndrome after axillary dissection. Am J Surg. 2001;181(5):434-439.

4. Leidenius M, Leppänen E, Krogerus L, von Smitten K. Motion restriction and axillary web syndrome after sentinel node biopsy and axillary clearance in breast cancer. Am J Surg. 2003;185(2):127-130.

5. Koehler LA, Hunter DW. Lymphspiration: the axillary web and its lymphatic origin. Lymphology. 2016;49(4):185-191.

6. Severeid K, Simpson J, Templeton B, York R, Hummel-Berry K, Leiserowitz A. Lymphatic cording among patients with breast cancer of melanoma referred to physical therapy. Rehabil Oncol. 2007;25(4):8-13.

7. O'Toole J, Miller CL, Specht MC, et al. Cording following treatment for breast cancer. Breast Cancer Res Treat. 2013;140(1):105-111.

8. Torres Lacomba M, Mayoral Del Moral O, Coperias Zazo JL, Yuste Sánchez MJ, Ferrandez JC, Zapico Goñi A. Axillary web syndrome after axillary dissection in breast cancer: a prospective study. Breast Cancer Res Treat. 2009;117(3):625-630.

9. Lauridsen MC, Overgaard M, Overgaard J, Hessov IB, Cristiansen P. Shoulder disability and late symptoms following surgery for early breast cancer. Acta Oncol. 2008;47(4):569-575.

10. Bergmann A, Mendes VV, de Almeida Dias R, do Amaral E Silva B, da Costa Leite Ferreira MG, Fabro EA. Incidence and risk factors for axillary web syndrome after breast cancer surgery. Breast Cancer Res Treat. 2012;131(3):987-992.

11. Baggi F, Nevola Teixeira LF, Gandini S, et al. Axillary web syndrome assessment using a self-assessment questionnaire: a prospective cohort study. Support Care Cancer. 2018;26(8):2801-2807.

12. Lewis PA, Cunningham JE. Dynamic angular petrissage as treatment for axillary web syndrome occurring after surgery for breast cancer: a case report. Int J Ther Massage Bodywork. 2016;9(2):28-37.

13. Figueira PVG, Haddad CAS, de Almeida Rizzi SKL, Facina G, Nazario ACP. Diagnosis of axillary web syndrome in patients after breast cancer surgery: epidemiology, risk factors, and clinical aspects: a prospective study. Am J Clin Oncol. 2018;41(10):992-996.

14. Wyrick SL, Waltke LJ, Ng AV. Physical therapy may promote resolution of lymphatic coding in breast cancer survivors. Rehabil Oncol. 2006;24(1):29-34.

15. Koehler LA. Axillary web syndrome and lymphedema, a new perspective. Natl Lymphedema Netw. 2006;18(3):9-10.

16. Reedijk M, Boerner S, Ghazarian D, McCready D. A case of axillary web syndrome with subcutaneous nodules following axillary surgery. Breast. 2006;15(3):4101-413.

17. Josenhans E. Physiotherapeutic treatment for axillary cord formation following breast cancer surgery. Pt Zeitschriftfür Physiother. 2007;59(9):868-878.

18. Wariss BR, Costa RM, Pereira AC, Koifman RJ, Bergmann A. Axillary web syndrome is not a risk factor for lymphoedema after 10 years of follow-up. Support Care Cancer. 2017;25(2):465-470.

19. Huang HC, Liu HH, Yin LY, Yeh CH, Tu CW, Yang CS. The upperlimb volumetric changes in breast cancer survivors with axillary web syndrome. Eur J Cancer Care (Engl). 2017;26(2):e12637.

20. Cho Y, Do J, Jung S, Kwon O, Jeon JY. Effects of a physical therapy program combined with manual lymphatic drainage on shoulder function, quality of life, lymphedema incidence, and pain in breast cancer patients with axillary web syndrome following axillary dissection. Support Care Cancer. 2016;24(5):2047-2057.

21. Furlan C, Matheus CN, Jales RM, Derchain S, Sarian LO. Vascular alterations in axillary and brachial vessels in patients with axillary web syndrome after breast cancer surgery. Lymphat Res Biol. 2018;16(3):287-293.

22. Koehler L. Axillary Web Syndrome Ongoing Medical Evaluation [dissertation]. Minnesota: University of Minnesota; 2013.

23. Salmon RJ, Berry MG, Hamelin JP. A novel treatment for postoperative mondor's disease: manual axial distraction. Breast J. 2009;15(4):381-384. 
24. Koehler LA, Hunter DW, Haddad TC, Blaes AH, Hirsch AT, Ludewig PM. Characterizing axillary web syndrome: ultrasonographic efficacy. Lymphology. 2014;47(4):156-163.

25. Leduc $\mathrm{O}$, Fumière $\mathrm{E}$, Banse $\mathrm{S}$, et al. Identification and description of the axillary web syndrome (AWS) by clinical signs, MRI and US imaging. Lymphology. 2014;47(4):164-176.

26. Rashtak S, Gamble GL, Gibson LE, Pittelkow MR. From furuncle to axillary web syndrome: shedding light on histopathology and pathogenesis. Dermatology. 2012;224(2):110-114.

27. Fourie WJ, Robb KA. Physiotherapy management of axillary web syndrome following breast cancer treatment: discussing the use of soft tissue techniques. Physiotherapy. 2009;95(4):314-320.
28. Kepics JM. Physical therapy treatment of axillary web syndrome. Rehabil Oncol. 2004;22(1):21-22.

29. Luz CMD, Deitos J, Siqueira TC, Palú M, Heck APF. Management of axillary web syndrome after breast cancer: evidence-based practice. Rev Bras Ginecol Obstet. 2017;39(11):632-639.

30. Zuther JE, Norton S. Lymphedema Management. The Comprehensive Guide for Practitioners. Vol 4. New York, NY: Thieme; 2017.

31. Thompson Buum HA, Koehler L, Tuttle TM. Venturing out on a limb: axillary web syndrome. Am J Med. 2017;130(5):e209-e210.

32. Cheville AL, Tchou J. Barriers to rehabilitation following surgery for primary breast cancer. J Surg Oncol. 2007;95(5):409-418.
Breast Cancer - Targets and Therapy

\section{Publish your work in this journal}

Breast Cancer - Targets and Therapy is an international, peerreviewed open access journal focusing on breast cancer research, identification of therapeutic targets and the optimal use of preventative and integrated treatment interventions to achieve improved outcomes, enhanced survival and quality of life for the cancer patient

\section{Dovepress}

The manuscript management system is completely online and includes a very quick and fair peer-review system, which is all easy to use. Visit http://www.dovepress.com/testimonials.php to read real quotes from published authors. 